Sharif University of Technology
Scientia Iranica
Transactions E: Industrial Engineering
http://scientiairanica.sharif.edu
IRA I ENTIA

\title{
A mathematical model for an integrated airline fleet assignment and crew scheduling problem solved by vibration damping optimization
}

\author{
A. Rashidi Komijan ${ }^{a, *}$, R. Tavakkoli-Moghaddam ${ }^{\mathrm{b}}$, and S.-A. Dalil ${ }^{\mathrm{c}}$ \\ a. Department of Industrial Engineering, Firoozkooh Branch, Islamic Azad University, Firoozkooh, Iran. \\ b. School of Industrial Engineering, College of Engineering, University of Tehran, Tehran, Iran. \\ c. Department of Industrial Engineering, South Tehran Branch, Islamic Azad University, Tehran, Iran.
}

Received 6 August 2018; received in revised form 16 June 2019; accepted 21 July 2019

KEYWORDS
Airline fleet
assignment;
Crew scheduling;
Integrated
mathematical model;
VDO algorithm;
Taguchi experimental
design.

\section{Introduction}

Airlines seek different methods to solve complex operational and technical airline problems and consider different operations research methods to tackle them [1]. The main critical problem here is airline planning

*. Corresponding author. Tel.: +9821 88347422

E-mail addresses: rashidi@azad.ac.ir (A. Rashidi Komijan); tavakoli@ut.ac.ir (R. Tavakkoli-Moghaddam);

ali.dalily@gmail.com (S.-A.Dalil)

doi: $10.24200 /$ sci.2019.51516.2230

\begin{abstract}
Fleet assignment and crew scheduling are the most complex airline optimization problems. In this research, an optimized crew pairing set was considered as input, and the crew was selected to be assigned to each certain crew pairing. This paper presents a novel model to integrate the fleet assignment and crew scheduling problems. In this model, closed routes for both crew and fleet are simultaneously taken into account. It also considers two consecutive flight legs and some other characteristics such as time lag, minimum permitted time lag, and maximum economic time. Moreover, a Vibration Damping Optimization (VDO) algorithm was introduced to find an appropriate solution to this problem during a reasonable time period. To this end, an experimental design based on the Taguchi method was taken into account. To evaluate the performance of the proposed VDO algorithm, four designed test problems were solved using the proposed VDO and the obtained results were compared with those from the optimal solution and Particle Swarm Optimization (PSO) algorithm. Then, 10 generated test problems on a large scale were solved using VDO and PSO. The results showed that in these four designed test problems, VDO and PSO solutions had $1.62 \%$ and $2.95 \%$ gaps in average with an optimal solution. Moreover, based on 10 generated test problems, on average, VDO, compared to PSO, could provide a $6.71 \%$ better solution in a shorter time span.
\end{abstract}

(C) 2021 Sharif University of Technology. All rights reserved. 


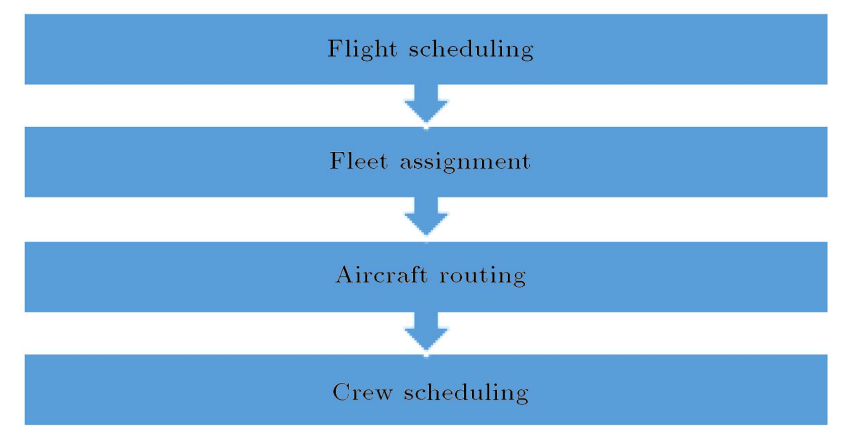

Figure 1. Sub-problems of the airline planning problem.

available aircraft of that type with a specific sequence while optimizing some objective functions under different operational and technical limitations [2]. Of note, the main focus of this section is put only on different fleet types, not any other specific aircrafts.

In aircraft routing problems, the schedule and route of each aircraft are determined in detail, considering maintenance requirements. The main objective of this section is to minimize aircraft costs with some considerations such as flight coverage, balance load of aircraft, and maintenance requirement [3].

Crew scheduling involves assigning the crew to designed flight legs. It can be applied to both cockpit and cabin crew according to the scheduling requirements. Aircraft routing and crew scheduling problems are usually taken into account after fleet assignment [4]. In the airline industry, the crew cost is the second highest cost after the fuel cost [1]. Crew scheduling includes two sub-problems: crew pairing and crew rostering problems [2]. In crew pairing, a certain sequence of flight legs generates candidate pairings. In crew rostering, each crew is assigned to certain pairings according to the rules and regulations. Different approaches have been employed so far to solve the above-mentioned sub-problems, such as column generation, benders decomposition, branch-and-price, and heuristic and meta-heuristic algorithms [2,4]. In this paper, flight scheduling is given and an integrated model is presented for crew scheduling and fleet assignment problems. The present study contributes to proposing a novel mathematical model for integrated fleet assignment and crew scheduling problems, considering closed routes for crew and fleets at the same time and applying a Vibration Damping Optimization (VDO) algorithm as the solution approach. Solving the model would lead to better crew and aircraft routing scheduling, thus determining that which pairings to select and assign to each crew. Further, solving the model specifies the flights each aircraft should do. The objective function of the model is to minimize the total cost that includes fleet operational, crew, deadhead flight, and aircraft-change costs. Having optimized the crew pairings, the model proposed by Ahmadbeygi et al. [5] was chosen and its generated pairings were considered as the input in this study.

Among the numerous studies integrating the subproblems of airline planning, the proposed model is the first one that has been solved by novel VDO algorithm. Moreover, Taguchi experimental method was employed to tune the algorithm parameters. Closed routes were also considered for crew and fleet simultaneously. In addition, the model utilized two consecutive flight legs and some other characteristics such as time lag, minimum permitted time lag, and maximum economic time. In order to prove the efficiency of the VDO algorithm, some numerical examples were developed and solved using General Algebraic Modeling System (GAMS), VDO, and Particle Swarm Optimization (PSO). The obtained results showed that in four designed test problems (small-sized problems), VDO and PSO solutions had $1.62 \%$ and $2.95 \%$ gaps on average with an optimal solution. Furthermore, based on 10 generated test problems (large-scale problems), VDO, compared to PSO, could offer a $6.71 \%$ better solution in a shorter time span, on average.

\section{Literature review}

Several airline planning and operation problems with different horizons have been addressed in the literature. The fleet assignment problem is usually formulated as linear programming, mixed-integer programming, or multi-commodity flow under operational and technical constraints $[1,2]$.

Sherali et al. [6] proposed different types of airline fleet assignment models considering both technical and operational constraints. They also presented different integrated fleet assignment problems with other sections of airline planning processes and surveyed different solution methods.

Sandhu and Klabjan [7] formulated the integrated fleet assignment and crew scheduling problems. They presented column generation, Lagrangian relaxation, and Benders decomposition methods as different solution approaches.

Papadakos [8] considered fleet assignment, aircraft maintenance routing, and crew scheduling problems in a single model and solved them using Benders decomposition and column generation methods. Gao et al. [9] presented an integrated mathematical model for airline fleet assignment and crew planning problems. Their proposed model can be employed to find optimal fleet assignment results and ensure robustness of crew planning and real-time operations. Ahmadbeygi et al. [5] presented a model to generate pairings based on some rules and regulations solved by exact methods such as branch and bound.

Weide et al. [10] solved the original aircraft routing and crew scheduling problems iteratively. They 
employed an iterative approach to model robustness. They also generated solutions that could add extra costs as penalties and cause robustness to stochastic variability in operation. Pilla et al. [11] referred to the fleet assignment problem as a stochastic programming model and employed a multivariate adaptive regression based on the cutting-plane method to solve it. Hu et al. [12] presented a model based on an approximate reduced time-band network for the aircraft routing problem considering transit passengers under disruption.

A Mixed Integer Programming (MIP) mathematical model presented by Cacchiani and SalazarGonzalez [13] could integrate fleet assignment, aircraft routing, and crew rostering problems and utilized a heuristic method for Canary Islands airlines. They proposed binary variables for potential aircraft and crew route. To obtain near-optimal solutions, they developed a column generation method. SalazarGonzalez [2] extended the model of Cacchiani and Salazar-Gonzalez [13] by considering a crew rostering problem and adding operational constraints such as classical capacity constraints and infeasible-path inequalities. They made an analogy between the crew rostering problem and multi-depot vehicle routing problem with the possibility of swapping drivers. They also regarded the vehicles as aircraft and the drivers as crew.

Diaz-Ramirez et al. [14] employed a heuristic method for aircraft routing and crew scheduling problems, considering one specific fleet, a single crew, and maintenance base. The crew scheduling problem was solved by a heuristic method to come up with an efficient, initial, and feasible solution as well as a labeling algorithm and column generation technique to solve the pricing problem. Finally, the model was formulated and compared with a traditional method. Cheng et al. [15] introduced the concept of flight operation risk assessment system for an airline and discussed the correlation between a risk factor and its sub components with fuzzy inference system. They also developed algorithms to identify the critical risk factors based on sensitivity of the risk factor and heuristic search.

Kasirzadeh et al. [4] conducted a survey for airline crew scheduling problems and solution methods. They also formulated the personalized crew scheduling problem for cockpit crew based on a classical set covering problem and solved it by a column generation method.

A non-linear model formulated by Gurkan et al. [16] was employed to integrate airline flight scheduling, fleet assignment, and aircraft routing problems. They also considered cruise speed control, fuel consumption, and $\mathrm{CO}_{2}$ emission and solved these problems using two heuristic methods in large-scale numerical cases. Dong et al. [17] formulated two models for flight scheduling and fleet assignment integration considering itinerary price elasticity.

Safak et al. [18] introduced a robust schedule design through cruise speed control and carbon emission. They employed a two-stage algorithm to decompose the problems and solve them sequentially.

Khaksar and Sheikholeslami [19] presented a method for airline delay prediction via machine learning algorithms based on data mining, random forest Bayesian classification, K-means clustering, and hybrid approach and calculated delay occurrence and magnitude in both Iran and USA networks.

A robust mathematical model was proposed by Jamili [20] to integrate flight scheduling, fleet assignment, and maintenance routing problems, and Simulated Annealing (SA) and PSO algorithms were employed to solve this model.

Safaei and Jardine [21] addressed the maintenance routing problem that could ensure enough time for maintenance operations. The presented model minimized the maintenance misalignment through an interactive method between the maintenance planning decisions and aircraft routing.

Chen et al. [22] formulated a mathematical programming model for aircraft leasing decisions about the aircraft requirements, budget, and debt-to-limit ratio. The objective function of their model was to minimize the costs. Kenan et al. [23] developed an integrated and stochastic model for flight scheduling, fleet assignment, and maintenance routing problems and solved them using column generation. Eltoukhy et al. [24] presented an operational maintenance routing model with a solution algorithm for finding suitable routing based on maintenance requirements. To this end, they regarded the flying time, number of take off/landing, and maintenance workforce as the constraints. They utilized their model in Egypt Air. Huu et al. [25] presented a multi-objective model to determine the aircraft departure routes and developed their model in Rotterdam and Amsterdam airports.

Ben Ahmed et al. [26] integrated aircraft routing and crew pairing and considered maintenance restriction in a robust mathematical model that could generate an elastic result for unpredictable disruption causing delays and flight cancelation. An integrated and stochastic model was proposed by Kenan et al. [27] for flight scheduling and fleet assignment problems. They could determine the flight demand through the stochastic parameter.

Based on the above literature, no research has been conducted so far that took into account the new variables and constraints for the proposed integrated model where the number of flight legs could be counted for each fleet. Moreover, no study has used the VDO algorithm for the integrated airline mathematical model after parameter tuning based on 
the Taguchi method. In this paper, the sequence of flight legs for a certain aircraft can be achieved and compared with that for a certain crew member under time lag and time limitation constraints. The aircraft routes were determined based on a set of sequences of assigned flight legs. In the meanwhile, the VDO algorithm was employed to find effective solutions for the mathematical model and the performance of VDO was compared with optimal results and PSO algorithm. The proposed model is capable of counting the number of flight legs. Furthermore, the sequence of flight legs for a certain aircraft can be achieved and compared with that for a certain crew member assigned to a specific pairing.

\section{Mathematical model}

This section discusses the integrated airline fleet assignment and crew scheduling problem for different pairings based on Integer Linear Programming (ILP) formulation. A certain pairing is a sequence of flight legs on different days that begins and ends at the base. Figure 2 shows four different generated pairings which are the closed sequences of flights after generating anonymous unassigned pairings based on time restrictions and cost limitations. Of note, these pairings are generated based on the crew pairing integer programming model proposed by Ahmadbeygi et al. [5]. In their model, first, each flight leg was assigned to the right aircraft type of each fleet based on the aircraft capacity and flying time limitation. Then, based on these generated pairings from the crew pairing model, each crew member should be assigned to a certain pairing. In this research, the cockpit crew members (e.g., pilots, co-pilots, flight engineers) were subject to these constraints and the cabin crew members (e.g., flight attendants and flight guards) were ignored. Given that each pairing takes place in three days at most, this approach can be used for the planning. In this respect, from now on, cockpit crew members will be referred to as crew in this research. Furthermore, each flight leg should be assigned only to one aircraft and one crew member. The time lag between two consecutive flight legs is one of the most significant subjects to be considered while assigning two consecutive flight legs to a specific aircraft. In other words, it should be applied when there is no aircraft change between two consecutive flight legs. This issue gains significance when the time lag should stand between two parameters, including the minimum permitted time (i.e., the least stopping time) that is needed between two consecutive flight legs and the maximum time (i.e., the maximum economic time) that is logical for an aircraft to stay in a specific airport. Given that aircraft change for each crew member may cause delays for the next flight legs (connection time based on the aircraft change may increase the possibility of delays), airline companies consider costs for these cases to reduce these delays by minimizing the costs of changing the aircrafts.

Aircraft routes comprise the sequence of the flight legs with arrival and departure times. Figure 3 shows the possible flight legs of four airports. For example, in this figure, the A-G-H flights sequence is a closed route for aircraft. Each flight leg has five characters, arrival time, departure time, origin, destination, and flight number. Time Away From Base (TAFB) is about the total time for a crew member that starts from the departure time of the first flight leg to the arrival time of the last flight leg with taking the sign in and sign out times into consideration.

Model assumptions are as follows:

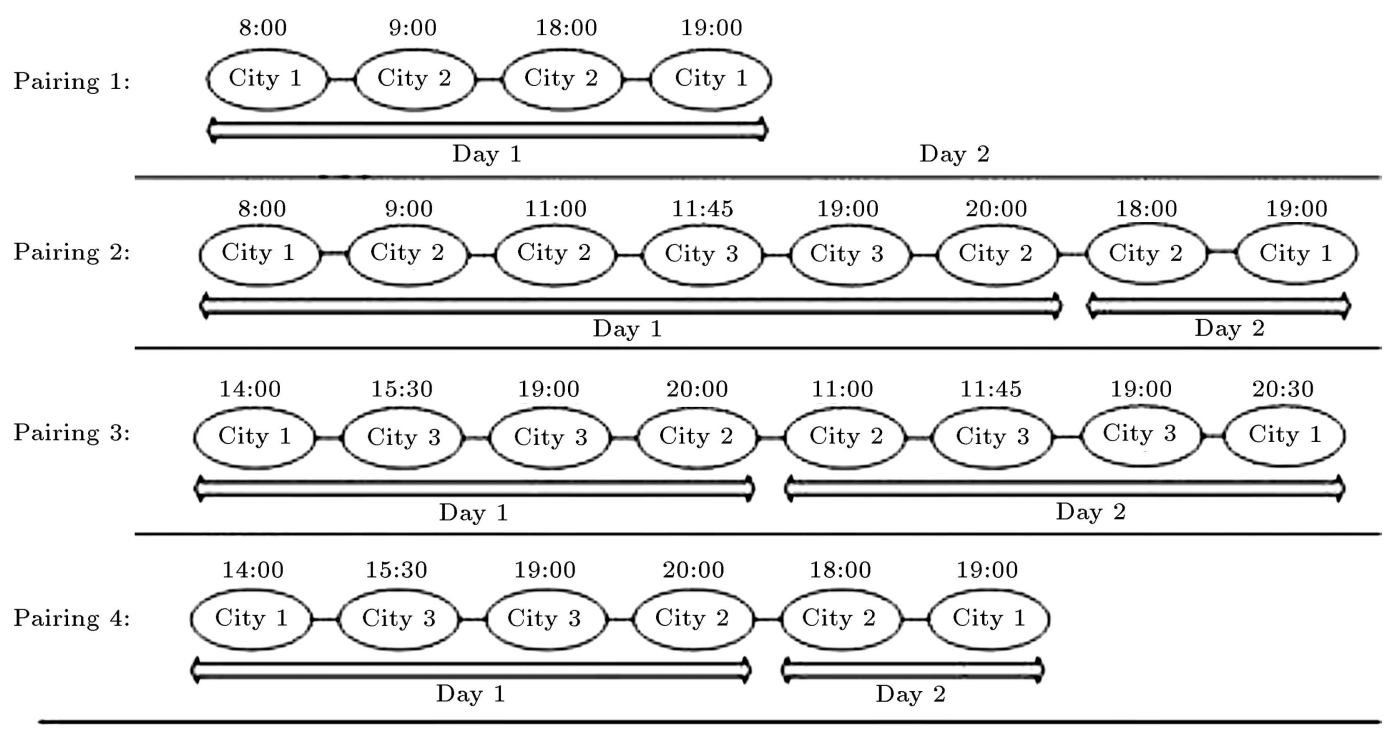

Figure 2. Examples of candidate pairings to be assigned to the crew. 


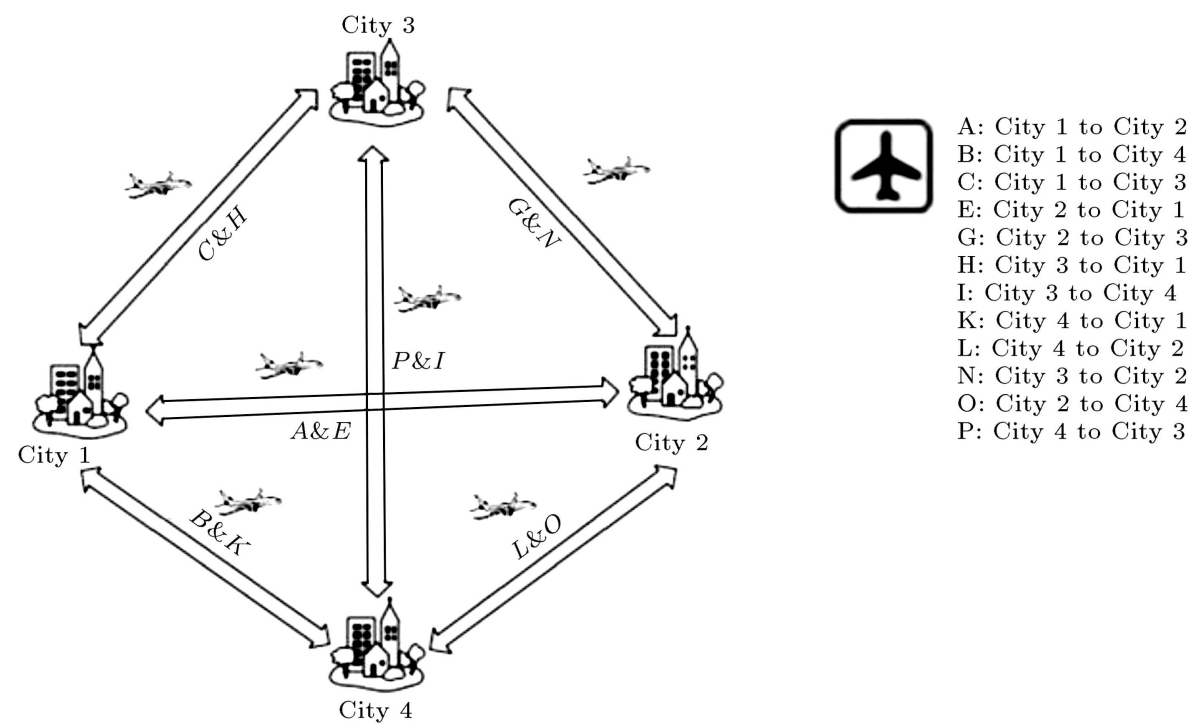

Figure 3. Typical sample of aircraft possible routes based on the flight legs.

- In the beginning of planning, several crew members and aircrafts are identified;

- Each aircraft can fly for the certain time period;

- Each flight leg should be assigned only to one crew member and one aircraft;

- Aircraft and crew routes are closed. In other words, each crew and aircraft start and end in the same base;

- There is no aircraft maintenance during the days of planning;

- There are no flight leg, maintenance, and inspection for aircraft between 11:00 pm and 7:00 am of the next day, and the daily rest time for crew members is considered in this time period.

Minimizing the total cost (i.e., the costs of fleet operational, crew, deadhead flight, and aircraft change) is considered as a significant objective function of this ILP model. Of note, this model considers aircraft types based on the fleet family type. The sets, indices, parameters, and variables are introduced in the following:

\section{Sets and indices:}

A Set of types of aircraft fleets available to be assigned to flight legs

$a \quad$ Aircraft index $\left(a=A_{1}, A_{2}, \cdots\right)$

$P \quad$ Set of possible pairings

$p \quad$ Pairing index $(p=1,2, \cdots)$

$F \quad$ Set of all flight legs

$F P_{f, f^{\prime}}^{p} \quad$ Set of consecutive flight legs $f, f^{\prime}$ of pairing $p$

$\theta_{1} \quad$ Set of flight legs with the same origin as the base $\theta_{2} \quad$ Set of flight legs with the same destination as the base

$\rho \quad$ Set of consecutive flight legs in which the destination of the first flight leg is the origin of the second flight

$f, f^{\prime} \quad$ Index of the designed flight leg $\left(f, f^{\prime}=A, B, \cdots\right)$

$N \quad$ Set of flight counter

$n \quad$ Index of flight counter $(n=1, \cdots, N)$

$C \quad$ Set of available crew members for the designed schedule

$C_{a} \quad$ Set of crew members able to fly with aircraft $a$

$c \quad$ Crew index $\left(c=C_{1}, C_{2}, \cdots\right)$

Parameters:

$F T_{f} \quad$ Flying time of flight leg $f$ per minute

Crew $C_{p, c} \quad$ Crew cost for each crew $c$ to be assigned to pairing $p$

Fleet $C_{f, a} \quad$ Fleet operational cost for aircraft a to be assigned to the flight leg $f$

Change $C_{f} \quad$ Cost of aircraft change for each flight $\operatorname{leg} f$

$D H_{f} \quad$ Cost of deadhead flight for each flight $\operatorname{leg} f$

$\operatorname{Lag}_{f, f^{\prime}} \quad$ Time lag between two consecutive flight legs $f$ and $f^{\prime}$ per minute

$\varphi_{a} \quad$ Maximum time restriction for each aircraft a per minute

LL Minimum permitted time lag for two consecutive flight legs per minute

UL Maximum economic time lag for two consecutive flight legs per minute 
M

Large positive number

\section{Decision variables:}

$X_{f, a, n} \quad 1$ if the flight leg $f$ is assigned to the aircraft $a$ in the $n$th flight leg; 0 , otherwise

$Y_{p, c} \quad 1$ if crew $c$ is assigned to the pairing $p$; 0 , otherwise

$Z_{f, c} \quad 1$ if flight leg $f$ in leading of crew $c$ has aircraft change; 0 , otherwise

The mathematical model for the integrated fleet assignment and crew scheduling problem is given below:

$$
\begin{aligned}
\min Z= & \sum_{c \in C} \sum_{p \in P} \operatorname{Crew}_{p, c} \cdot Y_{p, c} \\
& +\sum_{f \in F} \sum_{a \in A} \sum_{n \in N} \text { Fleet }_{f, a} \cdot X_{f, a, n} \\
& +\sum_{f \in F} \sum_{c \in C} \text { Change }_{f} \cdot Z_{f, c} \\
& +\sum_{f \in F}\left(\left(\sum_{a \in A} \sum_{n \in N} X_{f, a, n}\right)-1\right) \cdot D H_{f}
\end{aligned}
$$

s.t.:

$$
\begin{array}{ll}
\sum_{n \in N} \sum_{a \in A} X_{f, a, n}=1 & \forall f \in F, \\
\sum_{c \in C} Y_{p, c}=1 & \forall p \in P,
\end{array}
$$

$\sum_{n \in N} X_{f^{\prime}, a, n}+1-\sum_{n \in N} X_{f, a, n}+Y_{p, c} \leq Z_{f, c}+2$

$\forall\left(f^{\prime}, f\right) \in F P_{f^{\prime}, f}^{p}, \quad \forall a \in A, \quad \forall c \in C_{a}, \quad \forall p \in P$,

$$
\begin{aligned}
& L L-\left(2-\sum_{n \in N} X_{f, a, n}-\sum_{n \in N} X_{f^{\prime}, a, n}\right) \cdot M \leq \operatorname{lag}_{f, f^{\prime}} \\
& \leq U L+\left(2-\sum_{n \in N} X_{f, a, n}-\sum_{n \in N} X_{f^{\prime}, a, n}\right) \cdot M \\
& \forall\left(f, f^{\prime}\right) \in \rho, \quad \forall a \in A, \\
& X_{f^{\prime}, a, n} \leq \sum_{f:\left(f, f^{\prime}\right) \in \rho} X_{f, a, n-1} \\
& \forall f^{\prime} \in F-\theta_{1}, \quad \forall n>1, \quad n \in N, \quad \forall a \in A,(6) \\
& \sum_{f \in \theta_{1}} X_{f, a, 1} \leq 1 \quad \forall a \in A, \\
& \sum_{a \in A} X_{f, a, 1}=0 \quad \forall f \in F-\theta_{1},
\end{aligned}
$$

$$
\begin{aligned}
& \sum_{a \in A} X_{f, a, n}=0 \quad \forall f \in F-\theta_{2}, \quad n=N, \\
& X_{f^{\prime}, a, n} \leq \sum_{f:\left(f, f^{\prime}\right) \in \rho} X_{f, a, n-1} \\
& \forall f^{\prime} \in \theta_{1}, \quad \forall n>2, \quad n \in N, \quad \forall a \in A, \\
& \sum_{f \in F} \sum_{a \in A} X_{f, a, n} \leq 1 \quad \forall n \in N, \\
& T i c_{f} . X_{f, a, n} \leq C a p_{a} \\
& \forall f \in F, \quad \forall a \in A, \quad \forall n \in N, \\
& \sum_{n \in N} \sum_{f \in F} F T_{f} . X_{f, a, n} \leq \varphi_{a} \\
& \sum_{a \in A} \sum_{n \in N} X_{f, a, n} \leq \sum_{p \in P_{f}} \sum_{c \in C} Y_{p, c} \quad \forall a \in A, \\
& \sum_{p \in P} Y_{p, c} \leq 1 \\
& X_{f, a, n}, Y_{p, c}, Z_{f, c} \in\{0,1\} .
\end{aligned}
$$

The objective function (1) minimizes the total costs (i.e., the costs of fleet operational, crew, deadhead flight, and aircraft change). The first term of this objective function considers the related costs for the pairing $p$ assigned to the specific crew $c$. Each pairing has a different number of flight legs, in which the related costs for each pairing are different from those for the others. The second term is about fleet operational costs (e.g., the costs of fuel, preparation, stopping, landing, and taking-off). The third term presents the costs of aircraft change based on the flight legs. Finally, the last term considers deadhead flight leg costs for the flight legs appearing more than once in different selected pairings (i.e., if the flight leg $A$ exists in two different selected pairings $P_{1}=\{A, E\}$ and $P_{2}=\{A, G, H\}$, the flight leg $A$ is covered twice and one of them should take place as a deadhead flight leg and one of the assigned crew should be considered as a passenger on that flight leg).

Eq. (2) indicates that each flight leg should be assigned to one aircraft in one of the flight counters. Eq. (3) shows that some of the generated pairings are selected based on the fact that all flight legs should be covered by aircraft and crew to cover all of the flight legs. Eq. (4) ensures that if two consecutive flight legs of certain pairing assigned to a specific crew member with the same aircraft take place, no aircraft change may happen; otherwise, aircraft change would happen for these consecutive flight legs assigned to different aircrafts. Eq. (5) guarantees that the time 
lag between two consecutive flight legs is greater than the minimum permitted time, and these time lags are smaller than the maximum economic time. These two parameters are defined by the airline companies. The minimum permitted time is the least time required for an aircraft to be ready for the next flight. The maximum economic time is the longest time required according to the economic restriction of the companies.

Eq. (6) guarantees that if a flight leg with a different origin from base was selected as the second flight, there would be a flight leg, or legs, taking place before the mentioned flight leg. Eq. (7) lists the flight legs with origins the same as the base which can be selected as the first flight of aircraft. Eq. (8) shows that the flight legs with origins different from the base should not be chosen as the first flight of aircraft. In fact, all aircrafts should start from the base. Eq. (9) states that flight legs with a different destination from the base should not be chosen as the last flight of aircraft.

Eq. (10) indicates that if the flight legs with the same origins as the base are chosen as the third or next leg, there will be a flight leg, or legs, taking place before the mentioned flight leg. As mentioned earlier, the first flight leg should be chosen based on the $\theta_{1}$ set, an issue that needs to be taken into account in Eq. (7), and the second flight leg should be chosen based on the $\theta_{2}$ set; therefore, the third flight leg and the next can take place from the base again. This situation is possible based on the $\rho$ set.

Eq. (11) illustrates that aircraft transportation takes place when it is assigned to a flight leg. In other words, in each flight counter of an aircraft, only one flight leg may occur. Eq. (12) indicates that sold tickets of each flight leg should be harmonized with the aircraft capacity. In other words, sold tickets that must be equal to, or less than, the aircraft capacity are assigned to a specific flight leg. Eq. (13) indicates that the total flying time of each aircraft should not violate the maximum time restriction proposed by companies based on rules and regulations. Eq. (14) guarantees that a specific flight leg in any flight positioning (flight 1 , flight 2 , etc.) is chosen to be assigned to aircraft, and the pairing $\left(P_{f}\right)$ that includes that flight leg is selected.

Eq. (15) shows that in any scheduling period, each crew member is assigned to only one pairing. Adding this equation is worthy when a planner wants to select crew more fairly. Moreover, based on the assigned pairings and designed flight legs, TAFB is achievable for each assigned crew. Eq. (16) shows the types of the decision variables.

\section{Proposed VDO algorithm}

VDO algorithm is based on the vibration damping process [28]. To apply the VDO algorithm to the integrated airline fleet assignment and crew scheduling problem, several effective choices should be made.

\subsection{Solution representation}

A solution representation is divided into two parts. The first part presents a random sequence of the aircraft assigned to the sequence of flight legs. Of note, $\{f=$ $1,2, \cdots\}$ index is replaced with $\{f=A, B, C, \cdots\}$ index due to the large numbers of flight legs. The second part represents the random sequence of flight legs based on which each flight leg is assigned to available aircrafts. To elaborate the proposed algorithm, the following example is considered. Assume that five aircrafts are available and nine flight legs are designed for assignment. Tables 1 and 2 show $\theta_{1}, \theta_{2}$, and $\rho$ sets, respectively. Now, the sequence of flight legs and aircraft is presented in Table 3(a) and (b).

Then, based on the flight legs and aircraft sequence, flight legs are assigned to the aircraft. In case of the given example, first, aircraft 5 is selected and the flight legs with the value of 1 , from $\theta_{2}$, row of Table 1 would become candidates for assignment. According to the proposed $\theta_{2}$ set, the flight $\operatorname{legs} 4,8$, and 9 have the value of 1 . Therefore, for aircraft 5 , the flight leg that is in the fourth position (i.e., flight leg 3 ) of the flight leg sequence is first chosen for assignment. Now,

Table 1. $\theta_{1}$ and $\theta_{2}$ sets for the given example.

\begin{tabular}{cccccccccc}
\hline Flight legs & $\mathbf{1}$ & $\mathbf{2}$ & $\mathbf{3}$ & $\mathbf{4}$ & $\mathbf{5}$ & $\mathbf{6}$ & $\mathbf{7}$ & $\mathbf{8}$ & $\mathbf{9}$ \\
\hline $\boldsymbol{\theta}_{\mathbf{1}}$ & 1 & 1 & 1 & 0 & 0 & 0 & 0 & 0 & 0 \\
$\boldsymbol{\theta}_{\mathbf{2}}$ & 0 & 0 & 0 & 1 & 0 & 1 & 0 & 1 & 0 \\
\hline
\end{tabular}

Table 2. $\rho$ set for the given example.

\begin{tabular}{llllllllll}
\hline $\boldsymbol{\rho}$ & $\mathbf{1}$ & $\mathbf{2}$ & $\mathbf{3}$ & $\mathbf{4}$ & $\mathbf{5}$ & $\mathbf{6}$ & $\mathbf{7}$ & $\mathbf{8}$ & $\mathbf{9}$ \\
\hline $\mathbf{1}$ & 0 & 0 & 0 & 1 & 1 & 0 & 0 & 0 & 0 \\
$\mathbf{2}$ & 0 & 0 & 0 & 0 & 0 & 0 & 0 & 1 & 1 \\
$\mathbf{3}$ & 0 & 0 & 0 & 0 & 0 & 1 & 1 & 0 & 0 \\
$\mathbf{4}$ & 1 & 1 & 1 & 0 & 0 & 0 & 0 & 0 & 0 \\
$\mathbf{5}$ & 0 & 0 & 0 & 0 & 0 & 1 & 1 & 0 & 0 \\
$\mathbf{6}$ & 1 & 1 & 1 & 0 & 0 & 0 & 0 & 0 & 0 \\
$\mathbf{7}$ & 0 & 0 & 0 & 0 & 0 & 0 & 0 & 1 & 1 \\
$\mathbf{8}$ & 1 & 1 & 1 & 0 & 0 & 0 & 0 & 0 & 0 \\
$\mathbf{9}$ & 0 & 0 & 0 & 1 & 1 & 0 & 0 & 0 & 0 \\
\hline
\end{tabular}

Table 3. Solution representation for the sequence of flight legs and aircraft.

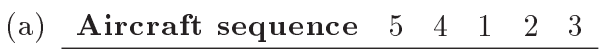
(b)

\begin{tabular}{llllllllll}
\hline Flights & 1 & 2 & 3 & 4 & 5 & 6 & 7 & 8 & 9 \\
Flight sequence & 8 & 7 & 6 & 3 & 9 & 5 & 4 & 2 & 1 \\
\hline
\end{tabular}


Table 4. Decoding the process of solution representation for the given example.

\begin{tabular}{|c|c|c|c|c|c|c|c|c|}
\hline \multirow{2}{*}{ (a) } & Flight & 1 & 2 & 3 & 4 & 5 & 6 & 78 \\
\hline & Flight sequence & 8 & 7 & 6 & 9 & 5 & 4 & 21 \\
\hline \multirow{2}{*}{ (b) } & Flight & 1 & 2 & 3 & 4 & 5 & 6 & \\
\hline & Flight sequence & 6 & 9 & 5 & 4 & 2 & 1 & \\
\hline \multirow{2}{*}{ (c) } & Flight & 1 & 2 & 3 & 4 & 5 & & \\
\hline & Flight sequence & 6 & 9 & 5 & 4 & 1 & & \\
\hline \multirow{2}{*}{ (d) } & Flight & 1 & 2 & 3 & 4 & & & \\
\hline & Flight sequence & 6 & 5 & 4 & 1 & & & \\
\hline
\end{tabular}

the sequence of flight legs is changed into Table 4(a). According to the changed sequence, it is time to check the destination $\left(\theta_{2}\right)$ for the last flight leg assigned to aircraft 5 . In the observations, the destination of the last flight leg is not the same as the base; therefore, another flight should be chosen for aircraft 5 . Based on the set $\rho$, the flight legs with the same origin as the destination of flight leg 7 are the candidate to be chosen, and based on the proposed example, flight legs 8 and 9 are the candidates. These two flight legs can occur consecutively. After the above discussion, the flight leg 8 is assigned to aircraft 5 as well. This flight will be deleted from the proposed sequence of flight legs and the new sequence will be obtained, as shown in Table 4(b). Another necessary assignment can be followed as other part of Table 4 . Based on decoding, the assignment of flight legs to different flight counters of the assigned aircraft is achievable (i.e., for aircraft 5 , the sequence of flight legs is flight 3 , flight 7 , flight 8 ; hence, flight 7 takes place as the second flight of aircraft 5). To assign these pairings to the crew members, the proposed VDO algorithm assigns the chosen pairings to the available crew members randomly. In other words, $Y_{p, c}$ decision variable matrices are randomly generated. Based on Eq. (4), the $Z_{f, c}$ decision variable matrices are achievable.

\subsection{Neighborhood structure}

A neighborhood structure was used for generating or developing a neighboring solution by incorporating some changes from the old solution to a new one. Different neighborhood structures were also applied to airline planning and operation problems. These neighborhood structures should generate only feasible solutions and eliminate all infeasible ones. In this paper, there are two types of operators that can generate neighborhoods. These operators are swap and reversion applied to the flight legs and aircraft sequences. While the swap involves changing the value of two positions, the reversion operator involves
Table 5. Sequences with swap operator.

\begin{tabular}{|c|c|c|c|c|c|c|c|c|c|}
\hline \multicolumn{10}{|c|}{ Current sequence for aircraft } \\
\hline Aircraft sequenc & & & & & 5 & 4 & 1 & 2 & 3 \\
\hline \multicolumn{10}{|c|}{ Current sequence for flight legs } \\
\hline Flight & 1 & 2 & 3 & 4 & 5 & 6 & 7 & 8 & 9 \\
\hline Flight sequence & 8 & 7 & 6 & 3 & 9 & 5 & 4 & 2 & 1 \\
\hline \multicolumn{10}{|c|}{ New sequence for aircraft } \\
\hline Aircraft sequenc & & & & & 5 & 2 & 1 & 4 & 3 \\
\hline \multicolumn{10}{|c|}{ New sequence for flight legs } \\
\hline Flight & 1 & 2 & 3 & 4 & 5 & 6 & 7 & 8 & 9 \\
\hline Flight sequence & 8 & 7 & 2 & 3 & 9 & 5 & 4 & 6 & 1 \\
\hline
\end{tabular}

Table 6. Sequences with the reversion operator.

\begin{tabular}{|c|c|c|c|c|c|c|c|c|c|}
\hline \multicolumn{10}{|c|}{ Current sequence for aircraft } \\
\hline Aircraft sequence & & & & & 5 & 4 & 1 & 2 & 3 \\
\hline \multicolumn{10}{|c|}{ Current sequence for flight legs } \\
\hline Flight & 1 & 2 & 3 & 4 & 5 & 6 & 7 & 8 & 9 \\
\hline Flight sequence & 8 & 7 & 6 & 3 & 9 & 5 & 4 & 2 & 1 \\
\hline \multicolumn{10}{|c|}{ New sequence for aircraft } \\
\hline Aircraft sequence & & & & & 5 & 3 & 2 & 1 & 4 \\
\hline \multicolumn{10}{|c|}{ New sequence for flight legs } \\
\hline Flight & 1 & 2 & 3 & 4 & 5 & 6 & 7 & 8 & 9 \\
\hline Flight sequence & 8 & 7 & 2 & 4 & 5 & 9 & 3 & 6 & 1 \\
\hline
\end{tabular}

arranging the positions from right to left for two candidate positions. Tables 5 and 6 show the process of swap and reversion operators.

\subsection{Steps of the proposed VDO algorithm}

Each iteration of the proposed VDO algorithm adds some random changes to the current solution which can generate a new solution in the neighborhood. Generation mechanisms define the neighborhood structure. As discussed in Section 4.2, the swap and reversion operators are chosen for the neighborhood structure. Once a new solution is generated, the corresponding changes in the cost function should be calculated to decide whether or not the new generated solution is acceptable. The steps of the proposed VDO algorithm are discussed in Figure 4. The stopping criterion for the proposed VDO algorithm can reach the supposed maximum number of iterations. Moreover, to achieve the suitable results, penalties are added to Eqs. (5), (12), and (13) to avoid violation in the algorithm.

\section{Parameter tuning}

One of the main concerns of creating each metaheuristic algorithm is to set suitable parameters to achieve acceptable performance for the proposed al- 


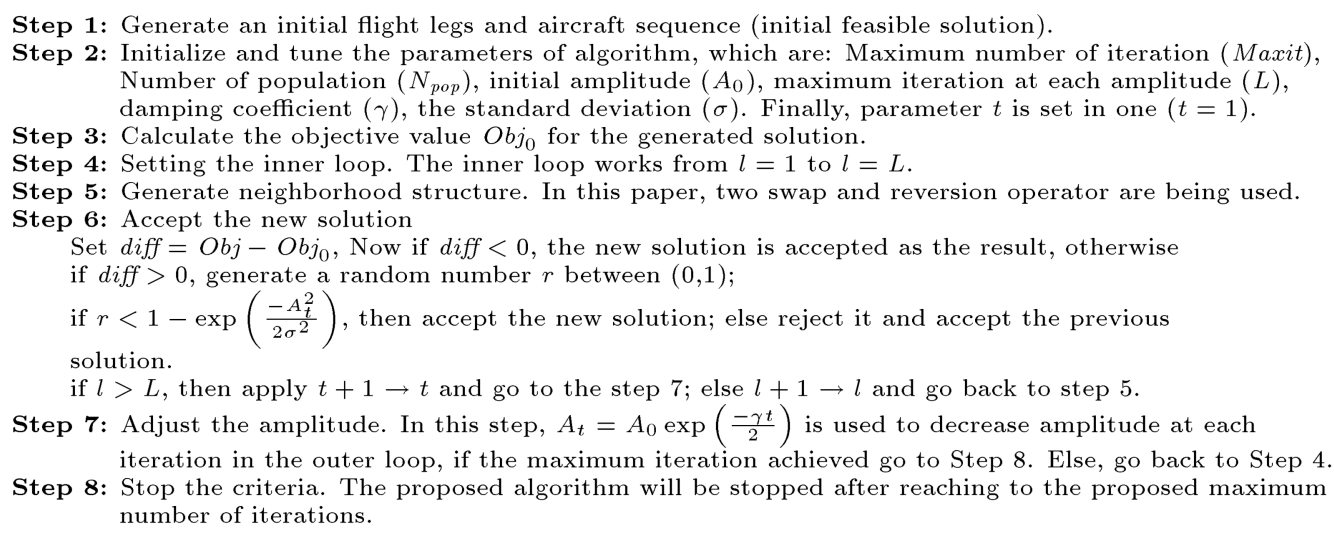

Figure 4. Steps of the proposed Vibration Damping Optimization (VDO) algorithms.

Table 7. Factors and levels of Vibration Damping Optimization (VDO) algorithm.

\begin{tabular}{ccccc}
\hline \multirow{2}{*}{ Factors } & \multirow{2}{*}{ Symbols } & \multicolumn{3}{c}{ Levels } \\
\cline { 3 - 5 } & & Low & Medium & \multicolumn{1}{c}{ High } \\
\hline \multirow{2}{*}{ Maxit } & $A$ & $A(L)=500$ & $A(M)=1000$ & $A(H)=1500$ \\
$N_{\text {pop }}$ & $B$ & $B(L)=50$ & $B(M)=100$ & $B(H)=200$ \\
$A_{0}$ & $C$ & $C(L)=10$ & $C(M)=20$ & $C(H)=30$ \\
$L$ & $D$ & $D(L)=4$ & $D(M)=6$ & $D(H)=8$ \\
$\gamma$ & $E$ & $E(L)=0.1$ & $E(M)=0.2$ & $E(H)=0.3$ \\
$\sigma$ & $F$ & $F(L)=0.6$ & $F(M)=0.7$ & $F(H)=0.8$ \\
\hline
\end{tabular}

gorithm. In this section, different parameter choices for the proposed VDO algorithm are studied. To tune the algorithm parameters, different methods for Design Of Experiments (DOE) should be introduced. One of these methods is the full factorial design used in different studies to design trials [28]. Although this method tests all possible combinations of factors, it is not logical to use it for a large number of factors due to the unacceptable costs and long run time.

Under such a condition, Taguchi experimental method is one of the DOE methods that employs orthogonal arrays to find suitable factors with a few numbers of experiments. Based on the Taguchi method, factors are divided into two parts, namely controllable and noise factors. According to this method, the inner and outer orthogonal arrays are replaced by the controllable and noise factors, respectively [28,29]. Based on the behavior of the noise factors, Taguchi method finds the best level for controllable factors while minimizing the corresponding effect of noise factors. Application of this method would bring about robustness to the factors [29]. Taguchi method transforms the repetitive data and causes variation. This repetitive data transformation is the Signal-to-Noise $(\mathrm{S} / \mathrm{N})$ ratio, clarifying the variation in the response variable. Both "signal" and "noise" are about the suitable and unsuitable values, respectively.

Taguchi method for parameter tuning is applied to gain suitable robustness for the proposed VDO algorithm. Based on parameters of the VDO algorithm, the control factors of the algorithm include $A_{0}, \sigma, \gamma$, $L$, Maxit, and $N_{\text {pop }}$. Table 7 presents three different levels, i.e., low, medium, and high, as denoted by $L$, $M$, and $H$, respectively, for factors.

To achieve the appropriate orthogonal array, six factors with three levels in the proposed algorithm are utilized. According to the standard table of orthogonal arrays, 27 different experiments, shown in Table 8, should be conducted. In this table, the control factors are shown at two and three levels, as shown in columns.

To obtain the desired experiment results, the proposed VDO algorithm is implemented in the MATLAB program on Windows 7 , installed on a PC with $1 \mathrm{~GB}$ RAM memory and $2.0 \mathrm{GHz}$. When the parameters are set as $A_{0}=30, L=8, \gamma=0.1$, and $\sigma=0.6$ with the maximum iteration of 1500 and the population number of 200, better algorithm robustness is achieved, as shown in Figure 5. Upon achieving the results based on different trials, the results of each trail are compared with the $\mathrm{S} / \mathrm{N}$ ratio, as shown in Figure 6 . 
Table 8. Orthogonal array L27 based on the proposed levels.

\begin{tabular}{|c|c|c|c|c|c|c|}
\hline \multirow{2}{*}{ Trails } & \multicolumn{6}{|c|}{ Different levels for factors } \\
\hline & $A$ & $B$ & $C$ & $D$ & $E$ & $F$ \\
\hline 1 & $A(L)$ & $B(L)$ & $C(L)$ & $D(L)$ & $E(L)$ & $F(L)$ \\
\hline 2 & $A(L)$ & $B(L)$ & $C(L)$ & $D(L)$ & $E(M)$ & $F(M)$ \\
\hline 3 & $A(L)$ & $B(L)$ & $C(L)$ & $D(L)$ & $E(H)$ & $F(H)$ \\
\hline 4 & $A(L)$ & $B(M)$ & $C(M)$ & $D(M)$ & $E(L)$ & $F(L)$ \\
\hline 5 & $A(L)$ & $B(M)$ & $C(M)$ & $D(M)$ & $E(M)$ & $F(M)$ \\
\hline 6 & $A(L)$ & $B(M)$ & $C(M)$ & $D(M)$ & $E(H)$ & $F(H)$ \\
\hline 7 & $A(L)$ & $B(H)$ & $C(H)$ & $D(H)$ & $E(L)$ & $F(L)$ \\
\hline 8 & $A(L)$ & $B(H)$ & $C(H)$ & $D(H)$ & $E(M)$ & $F(M)$ \\
\hline 9 & $A(L)$ & $B(H)$ & $C(H)$ & $D(H)$ & $E(H)$ & $F(H)$ \\
\hline 10 & $A(M)$ & $B(L)$ & $C(M)$ & $D(H)$ & $E(L)$ & $F(M)$ \\
\hline 11 & $A(M)$ & $B(L)$ & $C(M)$ & $D(H)$ & $E(M)$ & $F(H)$ \\
\hline 12 & $A(M)$ & $B(L)$ & $C(M)$ & $D(H)$ & $E(H)$ & $F(L)$ \\
\hline 13 & $A(M)$ & $B(M)$ & $C(H)$ & $D(L)$ & $E(L)$ & $F(M)$ \\
\hline 14 & $A(M)$ & $B(M)$ & $C(H)$ & $D(L)$ & $E(M)$ & $F(H)$ \\
\hline 15 & $A(M)$ & $B(M)$ & $C(H)$ & $D(L)$ & $E(H)$ & $F(L)$ \\
\hline 16 & $A(M)$ & $B(H)$ & $C(L)$ & $D(M)$ & $E(L)$ & $F(M)$ \\
\hline 17 & $A(M)$ & $B(H)$ & $C(L)$ & $D(M)$ & $E(M)$ & $F(H)$ \\
\hline 18 & $A(M)$ & $B(H)$ & $C(L)$ & $D(M)$ & $E(H)$ & $F(L)$ \\
\hline 19 & $A(H)$ & $B(L)$ & $C(H)$ & $D(M)$ & $E(L)$ & $F(H)$ \\
\hline 20 & $A(H)$ & $B(L)$ & $C(H)$ & $D(M)$ & $E(M)$ & $F(L)$ \\
\hline 21 & $A(H)$ & $B(L)$ & $C(H)$ & $D(M)$ & $E(H)$ & $F(M)$ \\
\hline 22 & $A(H)$ & $B(M)$ & $C(L)$ & $D(H)$ & $E(L)$ & $F(H)$ \\
\hline 23 & $A(H)$ & $B(M)$ & $C(L)$ & $D(H)$ & $E(M)$ & $F(L)$ \\
\hline 24 & $A(H)$ & $B(M)$ & $C(L)$ & $D(H)$ & $E(H)$ & $F(M)$ \\
\hline 25 & $A(H)$ & $B(H)$ & $C(M)$ & $D(L)$ & $E(L)$ & $F(H)$ \\
\hline 26 & $A(H)$ & $B(H)$ & $C(M)$ & $D(L)$ & $E(M)$ & $F(L)$ \\
\hline 27 & $A(H)$ & $B(H)$ & $C(M)$ & $D(L)$ & $E(H)$ & $F(M)$ \\
\hline
\end{tabular}

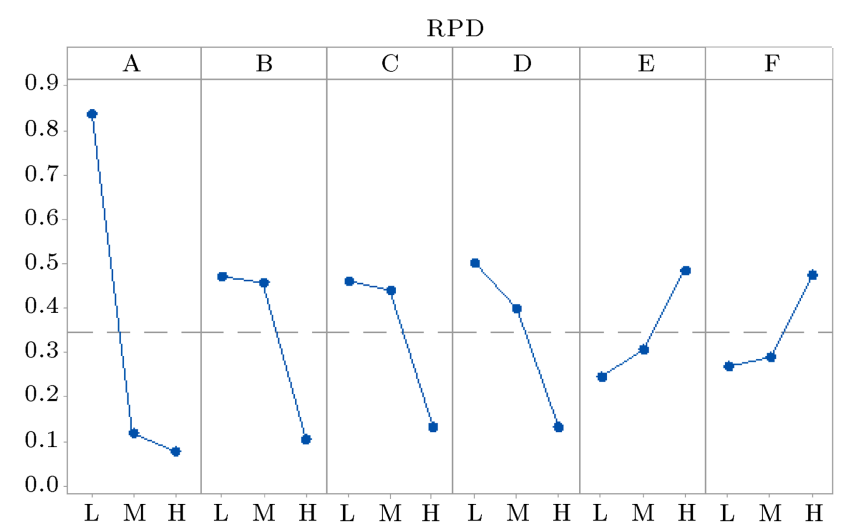

Figure 5. Relative Percentage Deviation (RPD) plot for factors at the proposed levels.

In order to adjust factors and compare trials, Relative Percentage Deviations (RPDs) were used for the Objective Function Value (OFV). The RPD is computed by:

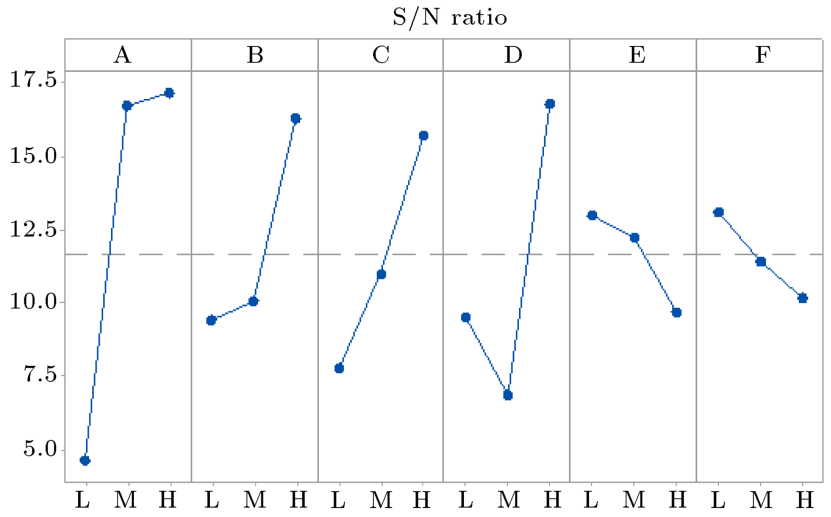

Figure 6. S/N plot for factors at the proposed levels.

$$
R P D=\frac{O b j_{a l g}-O b j_{\min }}{O b j_{\min }} \times 100,
$$

where $O b j_{a l g}$ stands for the OFV for each trail in a specific problem and $O b j_{\min }$ is the best OFV for the same problem. First, it should convert the OFV to RDPs and then, the RPD is calculated for each level. Figure 5 shows the RPD plot. Based on the RPD and $\mathrm{S} / \mathrm{N}$ ratio plots, the best levels of factors are as follows: $A(H), B(H), C(H), D(H), E(L)$, and $F(L)$.

\section{Computational results}

In this section, different test problems are proposed to evaluate the efficiency and effectiveness of the proposed VDO algorithm with the objective of finding good quality fleet and crew assignments. According to Section 5, Taguchi experimental method is used to find the best factor of parameters and their levels. Moreover, after ten runs, the best Objective Function Value $(\mathrm{OFV})$ is selected for each test problem. In this section, Table 9 illustrates the flight legs with their characteristics in Section 6.1. In this section, the proposed VDO algorithm is compared with the optimal method that is coded with the GAMS program. Table 10 compares the VDO and optimal results based on four designed test problems. In Section 6.2, based on Table 11, flight legs and pairings are randomly generated to create test problems. Table 12 shows the comparison between the proposed VDO and PSO performances according to 10 generated test problems.

\subsection{Comparison between the VDO, PSO algorithm, and optimal method}

In this section, four designed test problems are utilized and an airline crew pairing model presented by Ahmadbeygi et al. [5] is utilized to generate the candidate pairings. Table 9 shows the proposed flights and their characteristics.

According to Table 10, pairings are generated for each problem and the number of available aircrafts and crew members is given. Then, according to Section 2, 
Table 9. Proposed designed flight legs and their characteristics.

\begin{tabular}{cccccc}
\hline Flights & Origin & Destination & Departure time & Arrival time & Flying time \\
\hline A & City 1 & City 2 & $08: 00$ & $09: 00$ & 60 \\
B & City 1 & City 4 & $07: 00$ & $08: 20$ & 80 \\
C & City 1 & City 3 & $14: 00$ & $15: 30$ & 90 \\
E & City 2 & City 1 & $18: 00$ & $19: 00$ & 60 \\
G & City 2 & City 3 & $11: 00$ & $11: 45$ & 45 \\
H & City 3 & City 1 & $19: 00$ & $20: 30$ & 90 \\
I & City 3 & City 4 & $18: 00$ & $19: 05$ & 65 \\
K & City 4 & City 1 & $21: 00$ & $22: 20$ & 80 \\
L & City 4 & City 2 & $12: 00$ & $12: 40$ & 40 \\
N & City 3 & City 2 & $19: 00$ & $20: 00$ & 60 \\
O & City 2 & City 4 & $14: 00$ & $14: 40$ & 40 \\
P & City 4 & City 3 & $10: 00$ & $11: 05$ & 65 \\
\hline
\end{tabular}

each problem is formulated. Moreover, the second column of this table determines the designed flight legs and the third column shows the generated pairings based on the airline crew pairing model.

The fourth, fifth, and sixth columns represent the available aircraft, allowed number of flights, and available crew members, respectively. It should be noted that all four test problems are run 15 times and the mean of each test problem is shown in the table. Other columns present the best results, mean of results, and run time for the model based on the VDO and PSO algorithms and optimal method. The last two columns represent the gaps between different results of VDO and PSO algorithms and that of the optimal method embedded in GAMS. A review of the results in Table 10 showed that VDO and PSO algorithms as well as GAMS software with CPLEX solver were able to achieve suitable results for the designed test problems 1 to 4 . Moreover, according to the four designed test problems, the gaps and run times of two proposed methods versus optimal method are logical and VDO and PSO solutions have $1.62 \%$ and $2.95 \%$ gaps on average with optimal solutions, respectively.

\subsection{Proposed VDO algorithm versus PSO algorithm for large-scale test problems}

To test VDO algorithm and compare it with PSO algorithm, first, 10 test problems were generated in different sizes based on Table 11 with the parameters randomly generated.

Then, VDO and PSO algorithms were used for solving these problems based on the generated parameters and the proposed mathematical model. Table 12 shows the results of these problems. As expected, more flights and generated pairings would cause higher costs. All 10 test problems were run for 15 times, and the mean of each test problem is shown in the table. A review of the results in Table 12 showed that the VDO algorithm was able to solve large-scale problems. Moreover, the run times of VDO algorithm were suitable for different sizes of the problems and these times were smaller than six seconds and on average, VDO achieved a $6.71 \%$ better solution in a shorter time span than PSO. Of note, GAMS could not achieve any result for these 10 generated problems. According to the results, VDO outperformed PSO, but in case of some delays or technical disruptions during the planning, VDO parameters should be tuned again based on Taguchi experimental method.

\section{Conclusions and future research}

The present study aimed to propose a mathematical model for solving the integrated airline fleet assignment and crew scheduling problem. Generated pairings were considered as the input data for this problem based on the integer programming model of the airline crew pairing. In the proposed model, closed routes for crew and fleet were simultaneously considered. In addition, the model took into account two consecutive flight legs and some other characteristics such as time lag, minimum permitted time lag, and maximum economic time. To solve this model, a novel Vibration Damping Optimization (VDO) algorithm was employed and the obtained results were compared with Particle Swarm Optimization (PSO) and optimal solutions. The objective function of this model was to minimize the total cost (i.e., fleet and crew costs). In this regard, to find the best factor of parameters of the VDO algorithm, Taguchi design method was employed and 
Table 10. Comparison of Vibration Damping Optimization (VDO), Particle Swarm Optimization (PSO), and optimal algorithms.

\begin{tabular}{|c|c|c|c|c|c|c|c|c|c|c|c|c|c|c|c|}
\hline \multirow{2}{*}{ 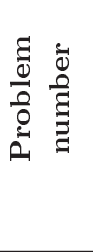 } & \multirow{2}{*}{ 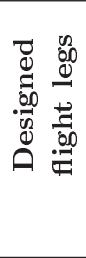 } & \multirow{2}{*}{ 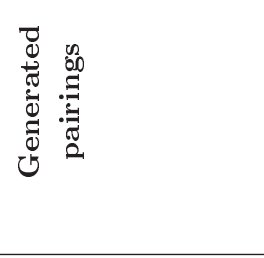 } & \multirow[b]{2}{*}{$\mathbf{A}$} & \multirow[b]{2}{*}{$\mathbf{N}$} & \multirow[b]{2}{*}{$\mathrm{C}$} & \multicolumn{3}{|c|}{$\begin{array}{c}\text { Proposed } \\
\text { VDO }\end{array}$} & \multicolumn{3}{|c|}{$\begin{array}{c}\text { Proposed } \\
\text { PSO }\end{array}$} & \multicolumn{2}{|c|}{ GAMS } & \multicolumn{2}{|c|}{$\begin{array}{c}\text { Algorithm } \\
\text { gaps }\end{array}$} \\
\hline & & & & & & $\begin{array}{l}\vec{D} \\
0 \\
0 \\
\vec{n} \\
0 \\
0\end{array}$ & 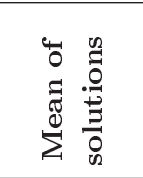 & 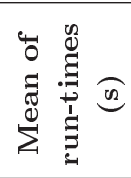 & $\begin{array}{l}\vec{r} \\
0 \\
0 \\
\vec{n} \\
0 \\
0 \\
0\end{array}$ & 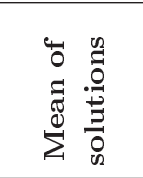 & 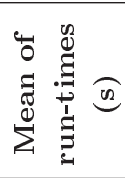 & 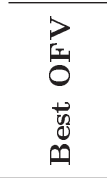 & 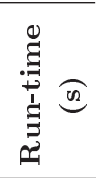 & 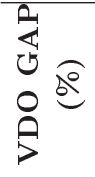 & $\begin{array}{l}0 \\
0 \\
0 \\
0 \\
0 \\
0\end{array}$ \\
\hline 1 & 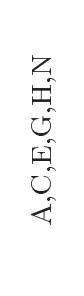 & $\begin{aligned} P_{1} & =\{A, E\} \\
P 2 & =\{A, G, H\} \\
P 3 & =\{C, H\} \\
P 4 & =\{C, N, E\} \\
P 5 & =\{A, G, N, E\} \\
P 6 & =\{C, N, G, H\}\end{aligned}$ & 3 & 3 & 4 & 259800 & 261156.5 & 0.5835 & 261200 & 270148.25 & 0.7613 & 258700 & 1.91 & 0.42 & 0.95 \\
\hline 2 & 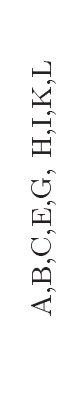 & $\begin{aligned} P_{1} & =\{A, E\} \\
P_{2} & =\{A, G, H\} \\
P_{3} & =\{A, G, I, K\} \\
P_{4} & =\{B, K\} \\
P_{5} & =\{B, L, G, H\} \\
P_{6} & =\{C, H\} \\
P_{7} & =\{C, I, K\} \\
P_{8} & =\{C, I, L, E\}\end{aligned}$ & 5 & 3 & 6 & 360800 & 360912.25 & 0.6246 & 362600 & 371132.5 & 0.8424 & 360400 & 1.838 & 0.11 & 0.61 \\
\hline 3 & D & $\begin{array}{l}P_{1}=\{A, E\} \\
P_{2}=\{A, G, H\} \\
P_{3}=\{A, G, I, K\} \\
P_{4}=\{B, K\} \\
P_{5}=\{B, L, G, H\} \\
P_{6}=\{C, H\} \\
P_{7}=\{C, I, K\} \\
P_{8}=\{C, I, L, E\}\end{array}$ & 5 & 5 & 6 & 242900 & 243255.35 & 0.6323 & 246800 & 248422.5 & 0.9113 & 234900 & 1.796 & 3.4 & 4.82 \\
\hline 4 & = & $\begin{array}{l}P_{1}=\{A, E\} \\
P_{2}=\{A, G, H\} \\
P_{3}=\{A, G, I, K\} \\
P_{4}=\{B, K\} \\
P_{5}=\{B, L, G, H\} \\
P_{6}=\{C, H\} \\
P_{7}=\{C, I, K\} \\
P_{8}=\{C, I, L, E\} \\
P_{9}=\{A, O, K\} \\
P_{10}=\{A, O, P, H\} \\
P_{11}=\{B, L, E\} \\
P_{12}=\{B, P, H\} \\
P_{13}=\{B, P, N, E\} \\
P_{14}=\{C, N, E\} \\
P_{15}=\{C, N, O, K\}\end{array}$ & 5 & 5 & 6 & 340600 & 340815.75 & 0.7542 & 351200 & 354225.5 & 1.474 & 332100 & 6.687 & 2.55 & 5.43 \\
\hline
\end{tabular}


Table 11. Generating parameters for large-scale problems.

\begin{tabular}{ll}
\hline Parameters & \multicolumn{1}{c}{ Random generation } \\
\hline$F T_{f}$ & Random number based on uniform distribution function $(45,90)$ \\
$\operatorname{Lag}_{f, f^{\prime}}$ & Based on generated sequence 0 or 1 \\
$\theta_{1}$ & Based on the origin of the generated flights 0 or 1 \\
$\theta_{2}$ & Based on the destination of the generated flights 0 or 1 \\
$C_{a}$ & Take 0 or 1 value randomly \\
$\rho$ & Based on the generated sequence 0 or 1 \\
$T_{i c_{f}}$ & Random number based on the uniform distribution function $(90,100)$ \\
$C a p_{a}$ & Generating random number based on the capacity of selected aircraft \\
$C h a n g e C_{f}$ & Random number based on the uniform distribution function $(1500,2500)$ \\
$\varphi$ & Random number based on the uniform distribution function $(500,700)$ \\
$F P_{f, f^{\prime}}^{p}$ & Generating random number based on the consecutive flights of each pairings \\
$P_{f}$ & Take 0 or 1 value based on the flights in each pairing \\
$C r e w C_{p, c}$ & Random number based on the uniform distribution function $(400,800)$ \\
$F l e e t C_{f, a}$ & Random number based on the uniform distribution function $(4500,5500)$ \\
$D H$ & Random number based on the uniform distribution function $(80,100)$ \\
\hline
\end{tabular}

Table 12. The proposed Vibration Damping Optimization (VDO) algorithm versus Particle Swarm Optimization (PSO) algorithm for different test problems.

\begin{tabular}{|c|c|c|c|c|c|c|c|c|}
\hline \multirow[t]{2}{*}{ Problem } & \multirow[t]{2}{*}{$\begin{array}{l}\text { Number of } \\
\text { flight legs }\end{array}$} & \multirow[t]{2}{*}{$\begin{array}{c}\text { Number of } \\
\text { generated } \\
\text { pairings }\end{array}$} & $\begin{array}{l}\text { Best } \\
\text { OFV }\end{array}$ & $\begin{array}{l}\text { Mean } \\
\text { OFV }\end{array}$ & $\begin{array}{l}\text { Mean of } \\
\text { run time } \\
(\mathrm{sec})\end{array}$ & $\begin{array}{l}\text { Best } \\
\text { OFV }\end{array}$ & $\begin{array}{l}\text { Mean } \\
\text { OFV }\end{array}$ & $\begin{array}{c}\text { Mean of } \\
\text { run time } \\
(\mathrm{sec})\end{array}$ \\
\hline & & & \multicolumn{3}{|c|}{ PSO } & \multicolumn{3}{|c|}{ VDO } \\
\hline 1 & 50 & 45 & 787370 & 793258.7 & 2.695 & 642750 & 653815.2 & 1.838 \\
\hline 2 & 60 & 55 & 831200 & 853516.5 & 2.828 & 761300 & 764625.8 & 1.946 \\
\hline 3 & 70 & 65 & 943580 & 987368.3 & 3.337 & 898850 & 901228.6 & 2.012 \\
\hline 4 & 90 & 75 & 1184840 & 1221134.6 & 3.752 & 1156950 & 1161124.4 & 2.324 \\
\hline 5 & 100 & 80 & 1202160 & 1214248.3 & 3.982 & 1198200 & 1200168.2 & 2.836 \\
\hline 6 & 120 & 100 & 1752200 & 1786402.2 & 4.442 & 1542600 & 1587772.5 & 3.218 \\
\hline 7 & 130 & 110 & 1812600 & 1868204.8 & 4.982 & 1649500 & 1652566.4 & 3.826 \\
\hline 8 & 140 & 120 & 1864260 & 1912268.5 & 5.112 & 1776400 & 1778504.8 & 4.322 \\
\hline 9 & 150 & 140 & 1988480 & 1994426.9 & 5.331 & 1928250 & 1930050.2 & 4.856 \\
\hline 10 & 200 & 150 & 2682200 & 2699860.3 & 5.973 & 2571000 & 2575608.6 & 5.768 \\
\hline
\end{tabular}

the robustness of this algorithms was improved by tuning the VDO parameters. Furthermore, the results of the proposed VDO and PSO with optimal solutions were compared, according to which VDO and PSO solutions had $1.62 \%$ and $2.95 \%$ gaps, respectively, with optimal solutions, on average. In order to examine the performance of the VDO algorithm, large-scale problems were generated and the performance of VDO was compared with that of PSO based on the generated test problems. According to the computational results, VDO algorithm could be utilized to optimize the given problems so that VDO could yield, on average, $6.71 \%$ better solution in a shorter time span than PSO.
It is suggested that flight scheduling be added as a new part of the integrated mathematical model or maintenance issues be applied to the aircraft routing problem. Of note, other constraints for fleets and crew such as different weight of aircraft and different type of crew should also be taken into account. Finally, to solve the given problem, developing a hybrid algorithm composed of VDO-PSO and VDO-GA is suggested.

\section{References}

1. Bazargan, M., Airline Operations and Scheduling, 2nd Edn., Ashgate Publishing Group, pp. 1-43 (2010). 
2. Salazar-Gonzalez, J.J. "Approaches to solve the fleet assignment, aircraft routing, crew pairing and crew rostering problems of a regional carrier", Omega, $\mathbf{4 3}$, pp. 71-82 (2014).

3. Basdere, M. and Bilge, U. "Operational aircraft maintenance routing problem with remaining time consideration", European Journal of Operational Research, 235, pp. 315-328 (2014).

4. Kasirzadeh, A., Saddoune, M., and Soumis, F. "Airline crew scheduling: models, algorithms, and data sets", Euro Journal of Transportation and Logistics, 6, pp. $1-27(2015)$.

5. AhmadBeygi, S., Cohn, A.M., and Weir, M. "An integer programming approach to generating airline crew pairings", Computers and Operation Research, 36(4), pp. 1284-1298 (2009).

6. Sherali, H.D., Bish, E.K., and Xiaomei, Z. "Airline fleet assignment concepts, models, and algorithms", European Journal of Operational Research, 172, pp. $1-30(2006)$.

7. Sandhu, R. and Klabjan, D. "Integrated airline fleeting and crew pairing decisions", Operation Research, 55, pp. 439-456 (2007).

8. Papadakos, N. "Integrated airline scheduling", Computer \& Operation Research, 36, pp. 176-195 (2009).

9. Gao, C., Johnson, E.L., and Smith, B.C. "Integrated airline fleet and crew robust planning", Transportation Science, 34(1), pp. 2-16 (2009).

10. Weide, O., Ryan, D., and Ehrgott, M. "An iterative approach to robust and integrated aircraft routing and crew scheduling", Computers and Operations Research, 37, pp. 833-844 (2010).

11. Pilla, V.L., Rosenberger, J.M., Chen, V., Engsuwan, N., and Siddappa, S. "A multivariate adaptive regression splines cutting plane approach for solving a twostage stochastic programming fleet assignment model", European Journal of Operational Research, 216, pp. 162-171 (2012).

12. Hu, Y., Xu, B., Bard, J.F., Chi, H., and Gao, M. "Optimization of multi-fleet aircraft routing considering passenger transiting under airline disruption", Computer \& Industrial Engineering, 80, pp. 1-31 (2014).

13. Cacchiani, V. and Salazar-Gonzalez, J.J. "A heuristic approach for an integrated fleet assignment, aircraft routing and crew pairing problem", Electronic Notes in Discrete Mathematics, 41, pp. 391-398 (2013).

14. Diaz-Ramirez, J., Ignacio Huertas, J., and Trigos, F. "Aircraft maintenance, routing and crew scheduling planning for airlines with a single fleet and a single maintenance and crew base", Computers \& Industrial Engineering, 75, pp. 68-78 (2014).
15. Cheng, C.B., Shyur, H.J., and Kou, Y.S. "Implementation of a flight operations risk assessment system and identification of critical risk factors", Scientia Iranica, Transactions E: Industrial Engineering, 21(6), pp. 2387-2398 (2014).

16. Gurkan, H., Gurel, S., and Akturk, M.S. "An integrated approach for airline scheduling, aircraft fleeting and routing with cruise speed control", Transportation Research Part C, 68, pp. 38-57 (2016).

17. Dong, Z., Yu, C., and Henry Lau, H.Y.K. "An integrated flight scheduling and fleet assignment method based on a discrete choice model", Computers \& Industrial Engineering, 98, pp. 195-210 (2016).

18. Safak, O., Gurel, S., and Akturk, M.S. "Integrated aircraft-path assignment and robust schedule design with cruise speed control", Computers and Operations Research, 84, pp. 127-145 (2017).

19. Khaksar, H. and Sheikholeslami, A. "Airline delay prediction by machine learning algorithms", Scientia Iranica, Transactions E: Industrial Engineering, 25, pp. 1-26 (2017).

20. Jamili, A. "A robust mathematical model and heuristic algorithms for integrated aircraft routing and scheduling with consideration of fleet assignment problem", Journal of Air Transport Management, 58, pp. 21-30 (2017).

21. Safaei, N. and Jardine, A.K.S. "Aircraft routing with generalized maintenance constraints", Omega, 80, pp. 1-12 (2017).

22. Chen, T.W., Huang, K., and Ardiansyah, M.N. "A mathematical programming model for aircraft leasing decisions", Journal of Air Transport Management, 69, pp. 15-25 (2018).

23. Kenan, N., Jebali, A., and Diabat, A. "The integrated aircraft routing problem with optional flights and delay considerations", Transportation Research Part E, 118, pp. 355-375 (2018).

24. Eltoukhy, E.E.A., Chan, T.S.F., Chung, S.H., and Niu, B. "A model with a solution algorithm for the operational aircraft maintenance routing problem", Computers \& Industrial Engineering, 120, pp. 346359 (2018).

25. Huu, V.H., Hartjes, S., Visser, H.G., and Curran, R. "Integrated design and allocation of optimal aircraft departure routes", Transportation Research Part D, 63, pp. 689-705 (2018).

26. Ben Ahmed, M., Zeghal Mansour, F., and Haouari, M. "Robust integrated maintenance aircraft routing and crew pairing", Journal of Air Transport Management, 73, pp. 15-31 (2018).

27. Kenan, N., Jebali, A., and Diabat, A. "An integrated flight scheduling and fleet assignment problem uncertainty", Computers \& Operations Research, 100, pp. 333-342 (2018). 
28. Mehdizadeh, E. and Tavakkoli-Moghaddam, R. "Vibration damping optimization", In Proc. of the Int. Conf. Operation Research, Augsburg, Germany (Sept. $3-5,2008)$.

29. Taguchi, G., Introduction to quality engineering, White Plains: Asian Productivity Organization/UNIPUN (1986).

\section{Biographies}

Alireza Rashidi-Komijan is an Associate Professor of Industrial Engineering. He received his $\mathrm{PhD}$ from Islamic Azad University in 2009. His major is Operations Research with focus on mathematical modeling, especially air transportation models. His research interest is large-scale scheduling problems.

Reza Tavakkoli-Moghaddam is a Professor of Industrial Engineering at the College of Engineering, University of Tehran, Iran. He obtained his PhD, $\mathrm{MSc}$, and BSc degrees in Industrial Engineering from the Swinburne University of Technology in Melbourne (1998), the University of Melbourne in Melbourne (1994), and the Iran University of Science and Technology in Tehran (1989), respectively. He serves as the Editor-in-Chief of the Journal of Industrial Engineering published by the University of Tehran and as the Editorial Board member of nine reputable academic journals. He is the recipient of the 2009 and 2011 Distinguished Researcher Awards and the 2010 and 2014 Distinguished Applied Research Awards at the University of Tehran, Iran. He has been selected as the National Iranian Distinguished Researcher in 2008 and 2010 by the MSRT (Ministry of Science, Research, and Technology) in Iran. He has obtained an outstanding rank as the top 1group since 2014. He also received the Order of Academic Palms Award as a distinguished educator and scholar for the insignia of Chevalier dans l'Ordre des Palmes Academiques by the Ministry of National Education of France in 2019. He has published 5 books, 32 book chapters, and more than 1000 journal and conference papers.

Seyed-Ali Dalil graduated with a BSc degree in Industrial Engineering/System Analysis from Qazvin Azad University, Iran and an MSc degree in Industrial Engineering from South Branch Islamic Azad University, Iran. He is currently a product expert at SHATEL Telecommunication Company, Iran. His research interests include airline planning and scheduling, airline fleet assignment, aircraft routing and airline crew scheduling problems, strategic product planning, project planning and controlling, and development of meta-heuristics for integrated airline planning problems. 